\title{
Current concept of the surgery-first orthognathic approach
}

\author{
Jong-Woo Choi ${ }^{1}$, Jang-Yeol Lee ${ }^{2}$ \\ ${ }^{1}$ Department of Plastic and Reconstructive Surgery, Asan Medical Center, University of Ulsan College of Medicine, Seoul; ${ }^{2}$ Smile Again \\ Orthodontic Center, Seoul, Korea
}

\begin{abstract}
Traditional orthognathic surgery has long been performed after presurgical orthodontic treatment. Despite some concerns, the surgery-first orthognathic approach (SFOA) or surgery-first approach (SFA) without presurgical orthodontic treatment has gradually gained popularity. In recent years, several articles dealing with the concepts of the SFA have been published worldwide. However, the SFA has not yet been standardized, and many surgeons use slightly different protocols and concepts. This review article discusses the beginning and evolution of the SFA and its current concepts, including some opinions based on the authors' clinical experiences over the last 15 years. According to recent investigations, the SFA could be applied effectively in several situations including class III malocclusion, class II malocclusion, and facial asymmetry. However, debate on the SFA continues and many issues remain to be resolved. This review article addresses the current issues regarding the SFOA, including its advantages and disadvantages, as well as its indications and contraindications. The authors summarize various aspects of the SFA and expect that this review article will help surgeons and orthodontists better understand the current status of the SFA.
\end{abstract}

Keywords Orthognathic surgery / Surgery first orthognathic approach / Surgery first approach / Orthodontic treatment
Correspondence: Jong-Woo Choi Department of Plastic and Reconstructive Surgery, Asan Medical Center, University of Ulsan College of Medicine, 88 Olympic-ro 43-gil, Songpa-gu, Seoul 05505, Korea Tel: +82-2-3010-3604 Fax: +82-2-476-7471

E-mail: pschoi@amc.seoul.kr

\section{INTRODUCTION}

The surgery-first approach (SFA) or the surgery-first orthognathic approach (SFOA) can be defined as an approach wherein orthognathic surgery is directly performed without presurgical orthodontic treatment, which was traditionally seen as a prerequisite for orthognathic surgery. Therefore, the SFA not only challenges the status quo, but also constitutes a new paradigm in craniomaxillofacial surgery.

Traditionally, in order to overcome postoperative occlusal instability, presurgical orthodontic treatment has long been re- garded as an essential step for obtaining successful outcomes in orthognathic procedures [1]. However, since the original cause of the dentofacial deformity being corrected by orthognathic surgery is a skeletal discrepancy, the question arises: why not first correct the skeletal discrepancy, which is the fundamental etiology of the dentofacial deformity? This sounds rational and logical. However, a problem is posed by the need to overcome postoperative occlusal instability.

There are three main approaches to solving this obstacle. First, South Korean groups seem to utilize the fact that the SFA is performed in the same direction as postsurgical orthodontic treat- 
ment [2]. Second, Japanese researchers such as the Sugawara group mostly rely upon active preoperative and postoperative tooth management, including cusp grinding and mini-screws [3]. Thirdly, researchers from Taiwan, such as the groups of Liu and Chen, suggested applying the SFA based on the regional acceleratory phenomenon (RAP) using corticotomy [4].

Although there are some controversies regarding who first suggested the SFA concept, it is clear that the earlier papers about this concept were mostly written in South Korea, as shown by searching for the first paper mentioning the SFA. In 2002, the Smile Again Orthodontic Group in South Korea published about the surgery-first concept in an article in the Korean Journal of Clinical Orthodontics using the term "functional orthognathic surgery," In this article, Oh and Son [5] clearly addressed and described the SFOA without presurgical orthodontic treatment, which is the fundamental basic concept underlying our current SFA (Fig. 1).

This paper reported that SFOA without presurgical orthodontic treatment could be possible based on a novel dental mock operation, including a process wherein presurgical orthodontic treatment was mimicked based on the separation of all of the teeth in the dental model. This early article showed several very successful surgical outcomes with our novel modern concept of SFA. Korean orthodontic groups such as the Smile Again Orthodontic Center have been using the SFA since 2001, and our institution, which has been collaborating with this group, has ap- plied the SFA for the last 15 years. Throughout this period, the authors have been suggesting that the SFA concepts should be adopted, and we have reported clinical outcomes from the SFA in clinical applications based on feasibility tests using dental model mock surgery in multiple serial publications.

This review addresses the current concept of the SFA and discusses controversial issues based on a review of the current literature and the authors' clinical experiences with the SFA for the last 15 years.

\section{DEFINITION AND EVOLUTION OF THE SFA}

The SFA is an orthognathic approach that consists of orthognathic surgery followed by postsurgical orthodontic treatment without any presurgical orthodontic treatment [6]. It is regarded as a paradigm shift confronting the traditional orthognathic approach. In the past, orthognathic surgery was sometimes performed without the proper presurgical orthodontic treatment before the establishment of the traditional modern protocol, which consists of presurgical orthodontic treatment for about 12-18 months, orthognathic surgery, and then postsurgical orthodontic treatment for roughly 6-12 months [7]. However, those attempts cannot be equated with the modern SFA concept.

Despite some controversies, the first paper on the SFA can be found in the Korean Journal of Clinical Orthodontics, in an article
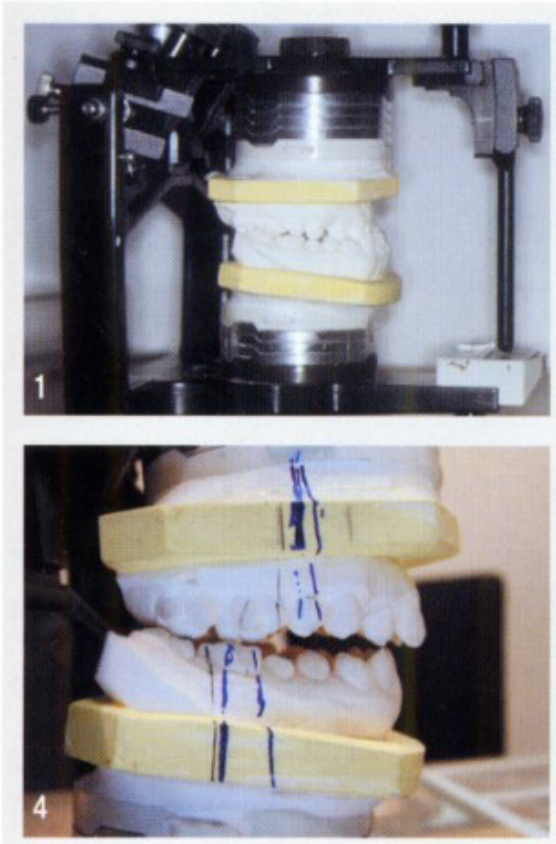
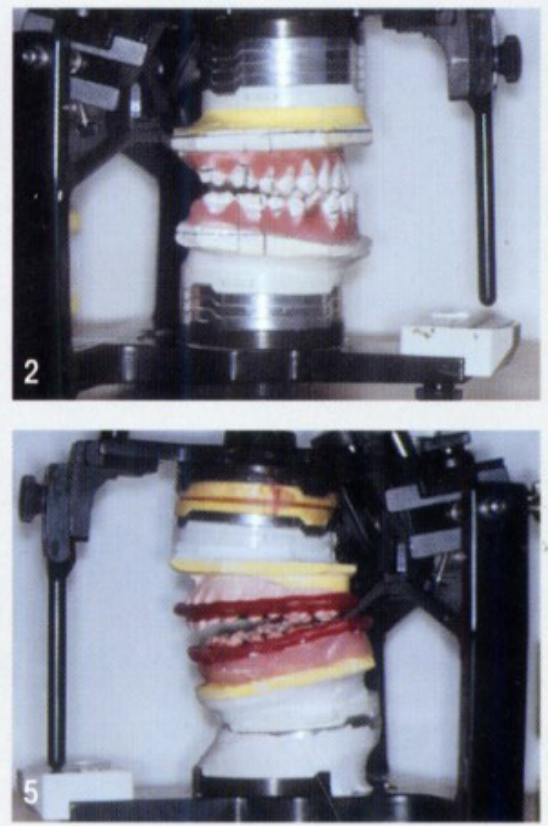
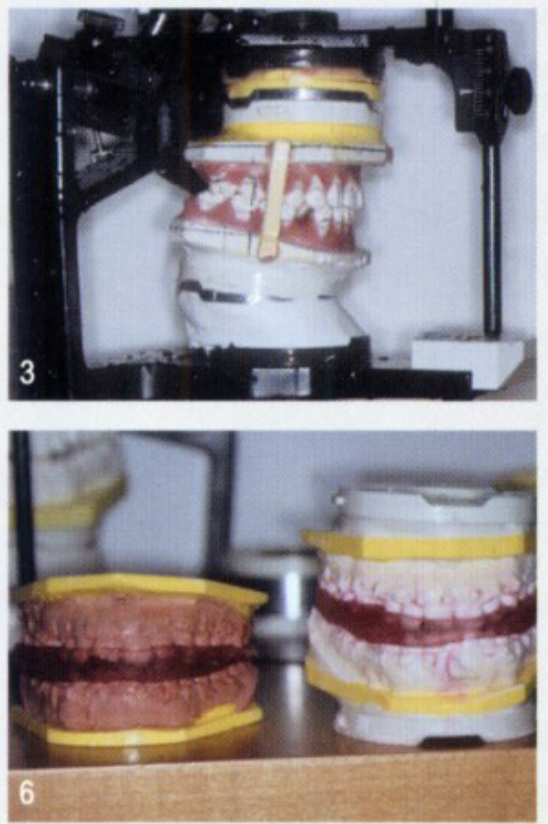

Fig. 1. Fundamental concept of the surgery-first orthognathic approach. This novel dental model set-up shows the surgery-first concept based on the separation of all of the teeth, mimicking the presurgical orthodontic treatment on the dental model, describing the surgery-first orthognathic approach without any presurgical orthodontic treatment. Reprinted from Oh et al. Korean J Clin Orthod 2002;1:32-9 [5]. 
published in 2002 [5]. This article first addressed the concept of the modern SFA using the term "functional orthognathic surgery." This concept, which was based on novel laboratory work, involved performing orthognathic surgery first, followed by postsurgical orthodontic treatment without presurgical orthodontic treatment. The clinical cases included in that articles were treated based on the separation of all of the teeth in a dental model to simulate the immediate postsurgical occlusal status without the application of any presurgical orthodontic treatment. The simulation of all of the teeth on the model allowed the surgeon or orthodontist to perform the SFA while skipping the traditional presurgical orthodontic treatment. This approach is still the fundamental basis of SFA clinical applications (Figs. 2, 3).

\section{BENEFITS AND DRAWBACKS OF THE SFA}

While traditional orthognathic surgery requires presurgical orthodontic treatment in order to achieve the goals listed below, these procedures are successfully completed after orthognathic surgery in the SFA.

\section{Conventional}

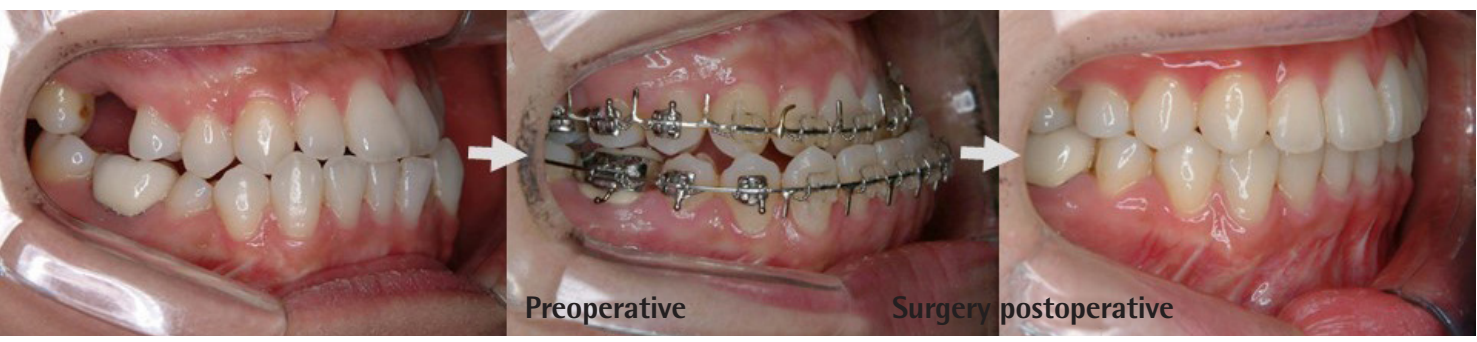

\section{Surgery-first approach}

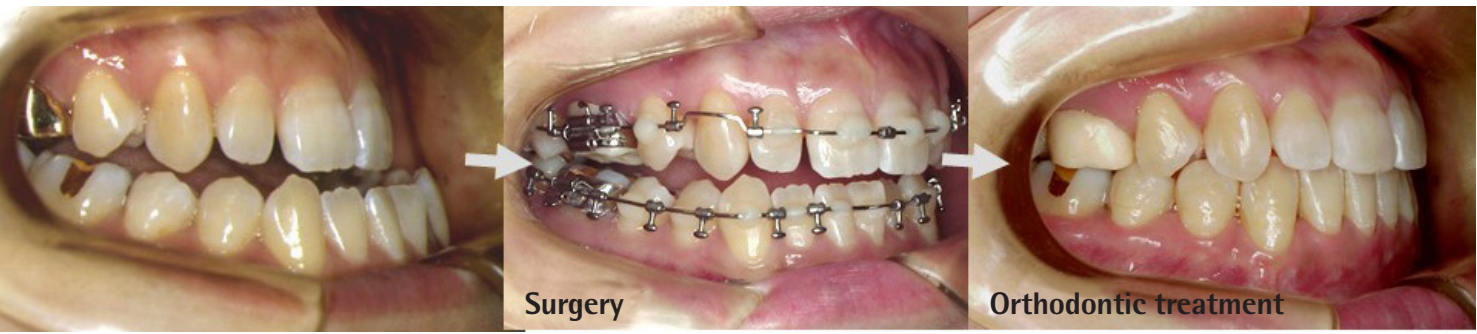

Fig. 2. Comparison between conventional and surgery first approach. The traditional orthognathic approach requires presurgical orthodontic treatment, such as leveling, decompensation, and arch coordination preoperatively, as shown above. Unlike the conventional approach, in the surgery-first approach (SFA), decompensation of the lower and upper teeth is not conducted preoperatively. Thus, the SFA inevitably leads to a pre-designed malocclusion status, which will be corrected in the postsurgical orthodontic treatment period. The direction of the surgical dental compensation is the same as that of the postsurgical orthodontic treatment.
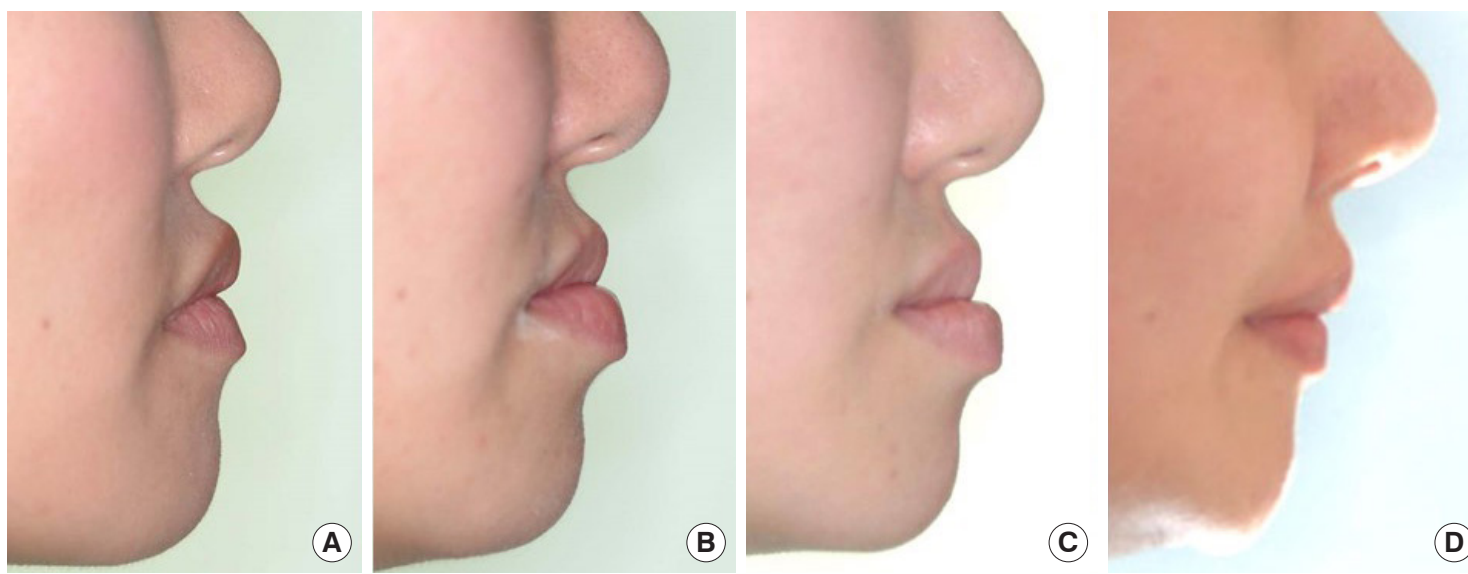

Fig. 3. Change of profile. Change in the soft tissue profile after the conventional orthognathic approach. (A) Initial, (B) 5 months preoperative orthognathic, (C) 10 months preoperative orthognathic, (D) after surgery. 


\section{Goals of preoperative orthodontic treatment for traditional orthognathic surgery}

- Elimination or reduction of dental compensation for the skeletal discrepancy

- Establishment of horizontal and vertical position of anterior teeth, canines, and posterior teeth

- Establishment of an arch form coordinating with each jaw

- Alignment to address the irregularity of teeth

\section{Advantages of the SFA}

- Direction of postsurgical orthodontics: identical with natural compensation

- Possibility of a shortened total treatment time

- No need for an aggravated gross appearance during the presurgical orthodontic period

- Minimal disturbance of the patient's social life

- Patient-oriented approach; early improvement of facial esthetics

- Efficient surgical/orthodontic timetable; enough postoperative time to manage skeletal and facial changes

- Early correction of sleep disorders

Tooth movement during presurgical orthodontic treatment is in the opposite direction of the functional compensation and has an adverse effect on the surrounding soft tissue during decompensation, which can prolong the presurgical orthodontic treatment. From the patient's perspective, presurgical orthodontic treatment can worsen facial esthetics, increase discomfort, and cause functional disturbances, which make the dental compensation more limited. On the contrary, in the SFA, the direction of dental decompensation after surgery is in the same direction as that of the dental and muscle adaptations to the surrounding new skeletal structures $[6,8]$. This is one of the main reasons why the total treatment time can be shortened by applying the SFA.

Another aspect regarding treatment time is the RAP, which can be maximized after surgery. Although some disagreements about the RAP exist, it appears quite obvious that tooth movement can be accelerated during the early stage after surgery. In the SFA, there is no need to aggravate the gross appearance of the patient during the presurgical orthodontic period. Early improvement of facial esthetics can improve patient satisfaction, and the SFA can minimize disturbances of the patient's social life. From the orthodontist's perspective, there is more time to observe the bone healing process and changes of bone segments after surgery; therefore, there is more flexibility for handling any possible skeletal relapses after surgery.

\section{Disadvantages of the SFA}

Some researchers have argued that the disadvantages of the SFA include less predictability of the mandibular counterclockwise rotation and difficulty in applying the SFA to severe transverse deficiency. The possible disadvantages of the SFA are summarized below.

- Time-consuming tooth set-up simulation for surgical occlusion

- More delicate and complicated surgical treatment objectives procedures

- Need for accurate and experienced decision-making regarding the possibility of SFA

- Complicated wire bending for the surgical archwire

- No possibility to extract third molars before surgery

- Possible need for an extended period of intermaxillary bony fixation

- Incomplete lip and facial profile immediately after surgery

- Chewing difficulties immediately after surgery due to incomplete occlusion

\section{AUTHORS STANDARD PROTOCOL FOR THE SFA}

Preoperatively, the model is mounted in the standard manner to assess the patient's occlusion (Fig. 4). In the model set-up, teeth that have already adapted to the skeletal discrepancy are simulated and reorganized into their predicted locations. In this process, each tooth in the model is analyzed, simulated, and separated, as would be done in real presurgical orthodontic treatment. Actual orthognathic surgery similar to the standard approach is then simulated. For example, in cases of class III malocclusion, maxillary impaction or advancement and mandibular setback processes are simulated. These processes indicate on the model the potential outcomes of occlusion after presurgical orthodontic treatment and orthognathic surgery. At this time, changing the position of the teeth on the dental model to the position before presurgical orthodontic treatment using the original teeth model yields a model reflecting the condition that would be obtained through orthognathic surgery without presurgical orthodontic treatment. This process is denoted as "surgical temporary occlusion." Unique magnetic mounting plates are designed to generate these changes in the dental model. Based on the simulated model surgery, intermediate and final wafers for orthognathic surgery without presurgical orthodontic treatment are prepared.

The remaining surgical steps of the SFA are similar to those of the standard approach. A LeFort I osteotomy with posterior maxillary impaction is performed, followed by mandibular set- 

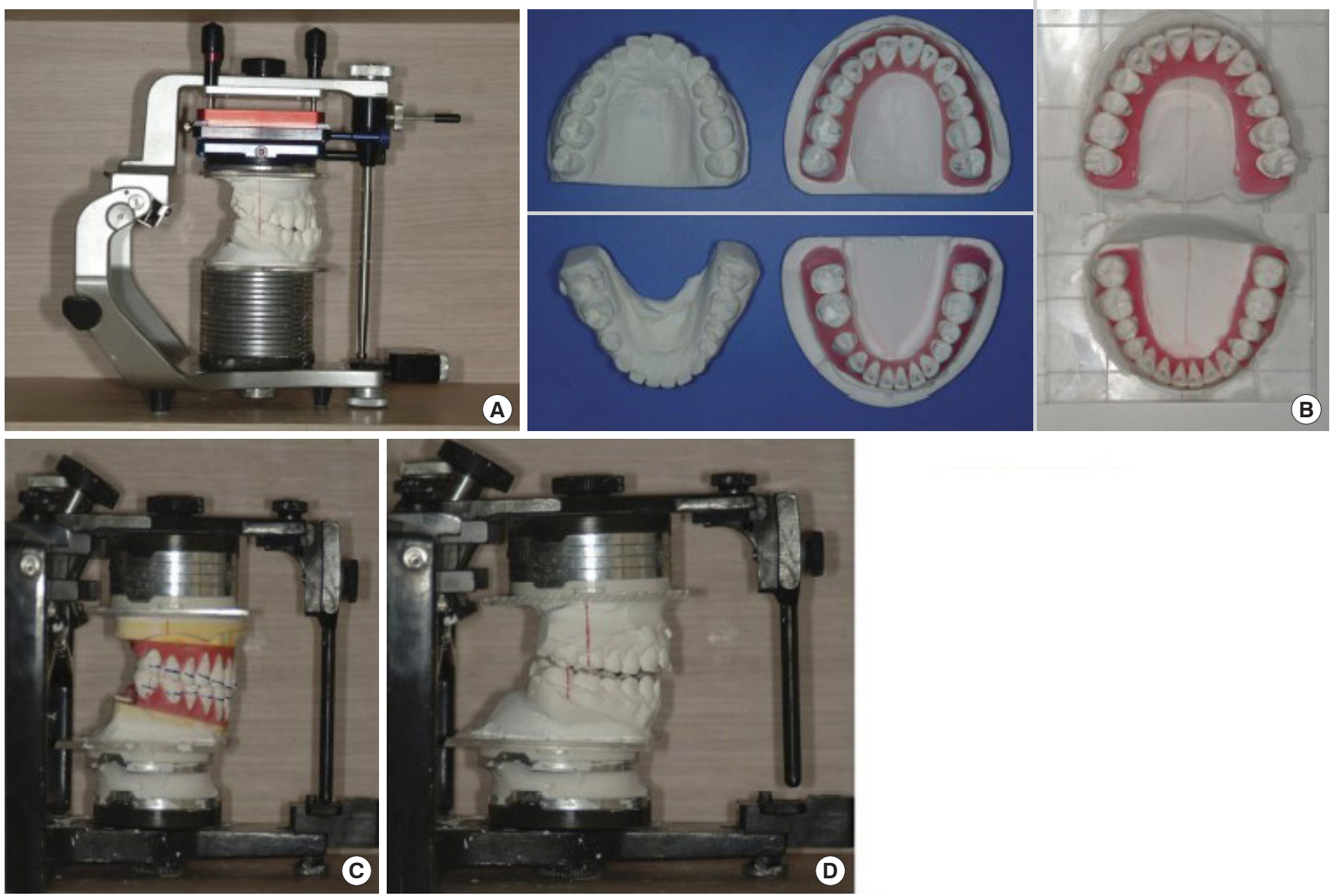

Fig. 4. Novel dental model mounting process. (A) Novel dental model mounting process. (B) For example, in cases of class III malocclusion, maxillary impaction or advancement and mandibular setback processes are simulated. These processes indicate on the model the outcome of possible occlusion after presurgical orthodontic treatment and orthognathic surgery. (C) At this time, if we change the position of the teeth on the dental model to the position before presurgical orthodontic treatment using the original teeth model, we obtain a model reflecting the condition of orthognathic surgery without presurgical orthodontics. (D) This process is referred to as "surgical temporary occlusion." Unique magnetic mounting plates are designed to produce these changes in the dental model. On the basis of the simulated model surgery, intermediate and final wafers for orthognathic surgery without presurgical orthodontic treatment are prepared.

back using sagittal split ramus osteotomy (SSRO) for clockwise rotation of the maxillomandibular complex, which appears to be ideal for many Asian class III patients with dentoalveolar protrusion. The proximal and distal mandibular segments are fixed using semirigid fixation with a miniplate.

\section{CONTROVERSIAL ISSUES IN THE SFA}

\section{Stability}

In general, the authors' clinical investigations based on 5 years of follow-up on average found skeletal stability in both the horizontal and vertical planes after the SFA. Patients who underwent traditional treatment and the SFA showed similar changes in skeletal landmarks, including the palatal plane angle; the Frankfort-mandibular plane angle; the mandibular plane-sellanasion line angle; the A-point to nasion-perpendicular line; the pogonion to nasion-perpendicular line; the A point, nasion, B point angle; facial convexity; and mandibular body length, but excluding the sella, nasion, B point angle; the ramus height; the upper incisor to stomion length; and occlusal plane to sella-nasion line angle. The two groups showed no significant differences in cephalometric skeletal landmarks, demonstrating the reliability of the SFA. For example, cephalometric landmarks representing the vertical component (e.g., the palatal and mandibular plane angles to the sella-nasion line) showed no interactions between the two groups at any time point $(\mathrm{P}=0.8272$ for the time interaction and $\mathrm{P}=0.2579$ for the group effect, respectively). This means that the amount of change in each group was almost the same for all periods and groups. Cephalometric landmarks representing horizontal skeletal components (e.g., the A point to nasion-perpendicular line and the pogonion to nasion-perpendicular line) likewise showed no significant differences between the two groups. These findings demonstrate that the ver- 
tical and horizontal skeletal patterns were not significantly different between the traditional approach and the SFA, confirming the reliability of the SFA $[9,10]$.

The position of the mandible showed the highest relapse rate. Horizontally, Ko et al. [4] reported a mean B-point relapse of $1.44 \mathrm{~mm}(12.46 \%)$ at a 1-year follow-up. Kim et al. [11] found average anterior relapses of $1.6 \mathrm{~mm}$ in patients who received conventional treatment and $2.4 \mathrm{~mm}$ in those who underwent the SFA, whereas Liao et al. [12] reported mild horizontal relapse in both groups. In our studies, vertical and skeletal stability were generally maintained, while the dental movement of the SFA surpassed that of the conventional approach $[9,10,13]$.

However, the relapses that many other authors mention appear to have resulted from mandibular autorotation, in our opinion. After resolving the occlusal interference, the mandible tends to be closed more completely. This may reflect simple autorotation instead of actual relapse, and should be investigated further. Regarding the choice between SSRO and intraoral vertical ramus osteotomy, the authors prefer SSRO in terms of the stability and minimization of postoperative physical therapy.

\section{Total treatment time}

Some authors have argued that the RAP could play a role in accelerating teeth movement in the postsurgical period because osteoblasts and osteocytes might be activated during the first several months postoperatively [13]. Therefore, some surgeons perform multiple corticotomies on the maxilla and mandibular bone to induce the RAP. In contrast, we did not perform any corticotomy procedures, but did observe that the total treatment time was shortened dramatically [7]. In our opinion, the fact that the direction of the postsurgical orthodontic movement is the same as that of natural compensation of teeth seems to play a much more important role in decreasing the overall treatment time. As a result of overcoming the temporary postoperative occlusional instability, postsurgical orthodontic treatment is more effective than presurgical orthodontic treatment that directs teeth movement opposite to that of the natural adaptational process of teeth. In addition, tooth extraction was found to be the factor with the greatest influence on the total treatment duration. Thus, regardless of the orthognathic approach, mobilization of the teeth may occur for some time after tooth extraction. Therefore, in order to achieve the maximal benefits of the SFA in terms of reducing the total treatment duration, SFA without tooth extraction would be a better treatment choice, if possible [8].

Despite the heterogeneity of publications, research on the SFA seems to consistently report a shorter treatment time. Overall, the mean treatment time was 14.2 months (range, 10.2-19.4 months) in surgery-first groups. The total treatment time was substantially longer in patients who received conventional treatment (mean, 20.16 months; range, 15.7-22.5 months) [14]. This may result from synergy between the orthodontic forces and the newly established adaptive forces from the lip and the tongue in the direction of tooth movement during postoperative orthodontic treatment, shortening the time to full compensation. The temporary decrease in muscle activity, bite force, and occlusal pressure for a few weeks after surgery could also facilitate this outcome [15]. Beyond the scope of the systematic review cited above, orthodontic treatment using the conventional approach has been reported to last from 15 to 24 months preoperatively and from 7 to 12 months postoperatively. It has been suggested that the orthodontist is the key decision-maker regarding this duration [16]. Likewise, we reported much shorter total treatment times than found in other reports of conventional orthodontic treatment in the literature, especially in nontooth extraction groups.

\section{Indications and contraindications Indications of the SFA}

If surgical occlusion in the SFA is conducted through simulation of postoperative orthodontic movement, the SFA could theoretically be applied to all surgical cases (Figs. 5-7). However, clinically, there could be several situations in which surgical correction through the SFA is inappropriate or would lead to instability. It would be reasonable to invert the proper indications by understanding these contraindications to the SFA. For example, based on our experiences, cleft-related dentofacial deformities and class II dentofacial deformities do not appear to be good indications for the SFA in general. Our team is very conservative in applying the SFA in these two groups. Concerning cleft-related dentofacial deformities, we do not feel that there is a need for the application of the SFA. Since we generally manage cleft patients from early childhood, we have abundant time for presurgical orthodontic treatment. For class II dentofacial deformities, the centric relation-centric occlusion discrepancy would be an obstacle. When there is a large centric relation-centric occlusion discrepancy, we are reluctant to apply the SFA because stabilization of the condyle is the top priority.

\section{Contraindications to the SFA}

The authors present the possible contraindications of the SFA, although some of the contraindications listed below could be overcome through accelerated presurgical orthodontic treatment (i.e., presurgical orthodontic treatment lasting for less than 3 months). 

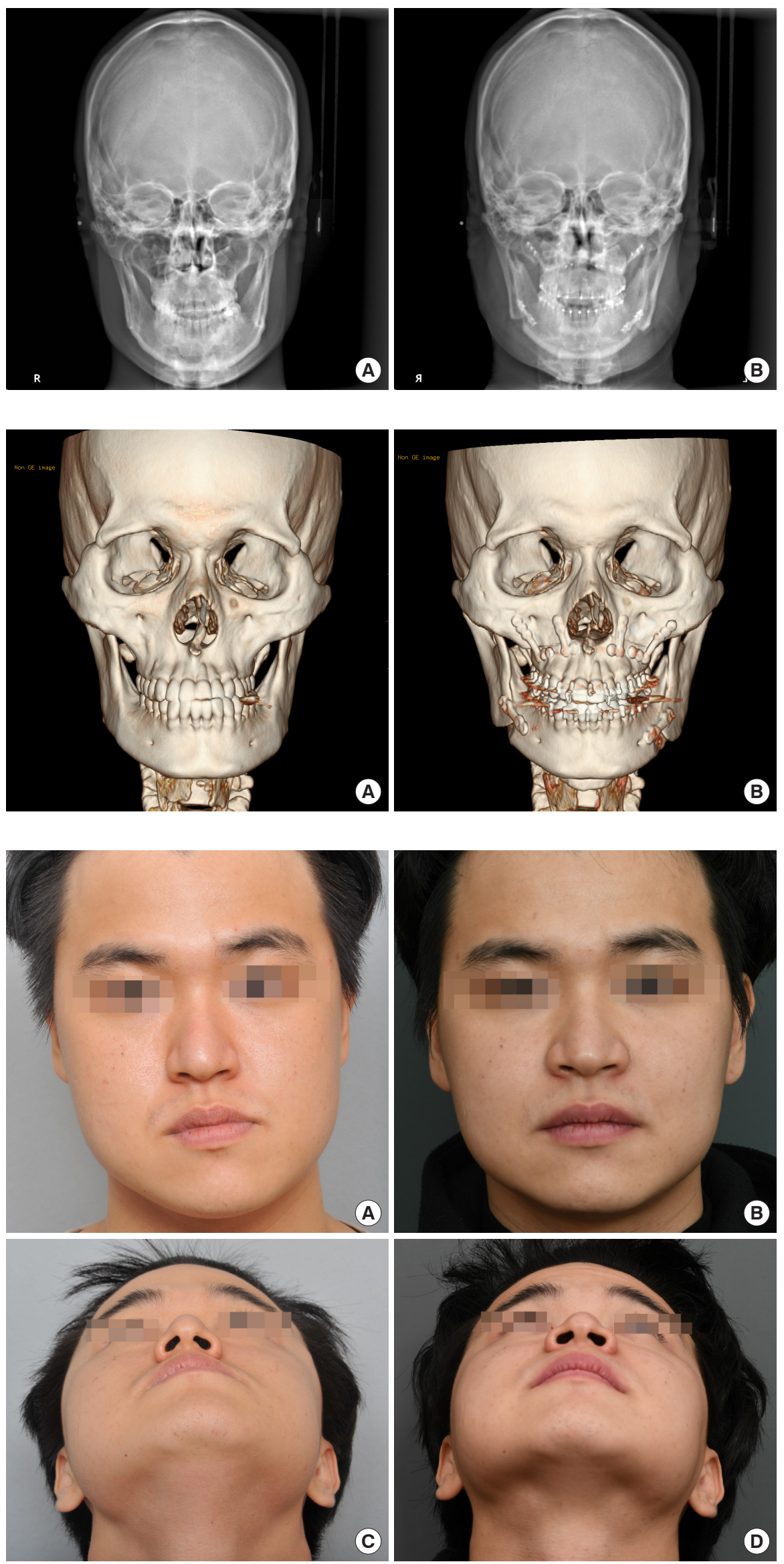

Fig. 5. Cephalometry. Surgical correction of facial asymmetry using the surgery-first approach without presurgical orthodontic treatment. (A) Preoperative view. (B) Postoperative view

Fig. 6. Three-dimensional computed tomography scan. Surgical correction of facial asymmetry using the surgery-first approach without presurgical orthodontic treatment. (A) Preoperative view. (B) Postoperative view

Fig. 7. Clinical photographs of the patient. Surgical correction of facial asymmetry using the surgery-first approach without presurgical orthodontic treatment. (A, C) Preoperative view. $(B, D)$ Postoperative view. 
Severe upper anterior crowding

A blocked upper lateral incisor on the palatal side may cause significant interference during surgical occlusion.

Severely compensated flared upper incisors

In patients with severely compensated flared upper incisors, it may be difficult to obtain aesthetic satisfaction immediately after surgery due to excessive overjet.

Excessively extruded upper second molars

Severe mandibular prognathism can cause excessive overeruption of the maxillary second molar because the maxillary second molar and the mandibular second molar do not occlude at all. If the amount of extrusion is too extreme, excessive interference may occur in the posterior surgical occlusion, which may compromise postoperative stability.

Disharmony of the upper and lower intercanine width Mandibular prognathism often results in functional displacement of the tongue, and when the tongue's position falls, spacing of the lower incisors occurs. This may result in discord between the upper and lower intercanine widths in the surgical occlusion, resulting in postoperative interference and bone instability.

Anterior crossbite after surgery

In cases of skeletal class II surgery, or even class III, a partial anterior crossbite occurs, and functional adaptation of the incisors may be hindered after surgery, which may make postoperative orthodontic treatment very difficult.

Asymmetrical transverse dental compensation in facial asymmetry

Severe horizontal asymmetry in patients with facial asymmetry may result in asymmetric transverse compensation of the left and right posterior teeth. In this case, surgical occlusion in the SFA can lead to unilateral posterior occlusion or excessive lateral compensation of the canine, which may result in insufficient asymmetry correction.

\section{DISCUSSION}

The SFA consists of orthognathic surgery followed by postsurgical orthodontic treatment without any presurgical orthodontic treatment. It is regarded as a paradigm shift confronting the traditional orthognathic approach. In the past, orthognathic surgery was often performed without the proper presurgical orthodontic treatment before the establishment of the traditional modern protocol consisting of presurgical orthodontic treatment for roughly 12-18 months, orthognathic surgery, and then postsurgical orthodontic treatment for approximately 6-12 months. However, the early approach cannot be equated with the modern SFA concept.

The modern concept of SFA is much more sophisticated. It should be based on a presurgical simulation and consideration of the following factors: whether occlusal instability can be overcome with postsurgical orthodontic treatment, including the active use of mini-screws; where the dentition should be moved postoperatively; and how far the dentition should be moved. These factors must be anticipated and planned in advance.

Regardless of how occlusal instability is measured after orthognathic surgery without presurgical orthodontic treatment, we believe that the modern concept of the SFA should be defined as a process involving simulation of the postsurgical occlusal status in advance. In recent years, many articles based on the surgery-first concept have been published, all of which propose different methodologies. In order to prevent the misuse of the surgery-first concept, we need to clarify the current concept and definition of the SFA. Doing so is the aim of this review article.

This paper addressed the current concept of the SFA and discussed controversial issues based on a review of the current literature and the authors' 15 years of clinical experience with the SFA.

\section{CONCLUSIONS}

The SFA without presurgical orthodontic treatment, using a unique presurgical simulation process on a dental model, is presented. Our findings indicate that the SFOA can achieve similar results in correcting dentofacial deformities as the orthodontic treatment-first approach. If applied appropriately, this novel approach could be an alternative to standard approaches in orthognathic surgery.

\section{NOTES}

\section{Conflict of interest}

Jong-Woo Choi is an editorial board member of the journal but did not involve in the peer reviewer selection, evaluation, or decision process of this article. No other potential conflicts of interest relevant to this article were reported.

\section{Patient consent}

The patients provided written informed consent for the publication and the use of their images. 


\section{Author contribution}

Conceptualization: JW Choi, JY Lee. Data curation: JY Lee. Formal analysis: JW Choi. Methodology: JW Choi, JY Lee. Project administration: JW Choi. Visualization: JW Choi. Writing - original draft: JW Choi. Writing - review \& editing: JW Choi.

\section{ORCID}

Jong-Woo Choi https://orcid.org/0000-0001-8047-9821

Jang-Yeol Lee https://orcid.org/0000-0003-3594-8750

\section{REFERENCES}

1. Obwegeser HL. Orthognathic surgery and a tale of how three procedures came to be: a letter to the next generations of surgeons. Clin Plast Surg 2007;34:331-55.

2. Choi JW, Lee JY, Yang SJ, et al. The reliability of a surgeryfirst orthognathic approach without presurgical orthodontic treatment for skeletal class III dentofacial deformity. Ann Plast Surg 2015;74:333-41.

3. Sugawara J, Aymach Z, Nagasaka DH, et al. "Surgery first" orthognathics to correct a skeletal class II malocclusion with an impinging bite. J Clin Orthod 2010;44:429-38.

4. Ko EW, Lin SC, Chen YR, et al. Skeletal and dental variables related to the stability of orthognathic surgery in skeletal class III malocclusion with a surgery-first approach. J Oral Maxillofac Surg 2013;71:e215-23.

5. Oh CO, Son HB. Functional orthognathic surgery (1). Korean J Clin Orthod 2002;1:32-9.

6. Choi JW, Bradley JP. Surgery first orthognathic approach without presurgical orthodontic treatment: questions and answers. J Craniofac Surg 2017;28:1330-3.

7. Jeong WS, Choi JW, Kim DY, et al. Can a surgery-first orthognathic approach reduce the total treatment time? Int J
Oral Maxillofac Surg 2017;46:473-82.

8. Jeong WS, Choi JW, Kim DY, et al. Can a surgery-first orthognathic approach reduce the total treatment time? Int J Oral Maxillofac Surg 2017;46:473-82.

9. Jeong WS, Lee JY, Choi JW. Large-scale study of long-term anteroposterior stability in a surgery-first orthognathic approach without presurgical orthodontic treatment. J Craniofac Surg 2017;28:2016-20.

10. Jeong WS, Lee JY, Choi JW. Large-scale study of long-term vertical skeletal stability in a surgery-first orthognathic approach without presurgical orthodontic treatment: part II. J Craniofac Surg 2018;29:953-8.

11. Kim JY, Jung HD, Kim SY, et al. Postoperative stability for surgery-first approach using intraoral vertical ramus osteotomy: 12 month follow-up. Br J Oral Maxillofac Surg 2014; 52:539-44.

12. Liao YF, Chen YF, Yao CF, et al. Long-term outcomes of bimaxillary surgery for treatment of asymmetric skeletal class III deformity using surgery-first approach. Clin Oral Investig 2019;23:1685-93.

13. Yaffe A, Fine N, Binderman I. Regional accelerated phenomenon in the mandible following mucoperiosteal flap surgery.J Periodontol 1994;65:79-83.

14. Peiro-Guijarro MA, Guijarro-Martinez R, Hernandez-Alfaro F. Surgery first in orthognathic surgery: a systematic review of the literature. Am J Orthod Dentofacial Orthop 2016; 149:448-62.

15. Uribe F, Adabi S, Janakiraman N, et al. Treatment duration and factors associated with the surgery-first approach: a two-center study. Prog Orthod 2015;16:29.

16. Luther F, Morris DO, Hart C. Orthodontic preparation for orthognathic surgery: how long does it take and why? A retrospective study. Br J Oral Maxillofac Surg 2003;41:401-6. 